\title{
Actions of human telomerase beyond telomeres
}

\author{
Yusheng Cong ${ }^{1}$, Jerry W Shay ${ }^{2}$ \\ ${ }^{1}$ Key laboratory for Cell Proliferation and Regulation Biology of the Ministry of Education, Institute of Cell Biology, Beijing Normal \\ University, 19 Xin Jie Kou Wai Avenue, Beijing 100875, China, ${ }^{2}$ Department of Cell Biology, UT Southwestern Medical Center, 5323 \\ Harry Hines Blvd, Dallas, TX 75390-9039, USA
}

Telomerase has fundamental roles in bypassing cellular aging and in cancer progression by maintaining telomere homeostasis and integrity. However, recent studies have led some investigators to suggest novel biochemical properties of telomerase in several essential cell signaling pathways without apparent involvement of its well established function in telomere maintenance. These observations may further enhance our understanding of the molecular actions of telomerase in aging and cancer. This review will provide an update on the extracurricular activities of telomerase in apoptosis, DNA repair, stem cell function, and in the regulation of gene expression.

Keywords: aging, cancer, immortalization, apoptosis, DNA damage response

Cell Research (2008) 18:725-732. doi: 10.1038/cr.2008.74; published online 24 June 2008

\section{Introduction}

Special properties of linear chromosome ends, telomeres, that protect them from end-to-end fusions in eukaryotic organisms, had been described long before the discovery of the DNA double helix structure $[1,2]$. The telomere is a special functional complex consisting of tandem repeated DNA sequences and its associated proteins [3]. The synthesis of telomere sequences is mainly achieved by the cellular reverse transcriptase telomerase, an RNA dependent DNA polymerase that adds telomeric DNA onto telomeres [4]. In humans, telomerase has a minimum of two essential components: the functional RNA component ( $h T R$ or $h T E R C)$ that serves as a template for telomeric DNA synthesis; and the catalytic protein component with reverse transcriptase activity (hTERT) that adds the telomeric repeats onto the end of chromosome $[5,6]$.

Most human somatic cells have undetectable telomerase activity due to transcriptional repression of the catalytic subunit hTERT during early embryonic development. Consequently human somatic cells lose telomeric DNA in cell culture by 50-200 base pairs after each round of replication. Thus, normal somatic cells without telomerase activity can only proliferate a limited number of times in culture. Telomere shortening also occurs with increased age in vivo, even

Correspondence: Yusheng Cong ${ }^{\mathrm{a}}$, Jerry W Shay ${ }^{\mathrm{b}}$

${ }^{a}$ Tel: 86-010-5880-2030; E-mail: yscong@bnu.edu.cn

bTel: 214-648-3282; E-mail: jerry.shay@utsouthwestern.edu though it remains to be determined how important telomere shortening is in overall organism aging. It is believed that when one or more telomeres become critically shortened, the protective function of the telomere is compromised; the dysfunctional telomere(s) is recognized as damaged DNA, which triggers a permanent growth arrest known as replicative senescence. The senescence phenotype can also be induced by insults including oxidative stress, overexpression of certain oncogenes, and DNA damage signals. This type of senescence was originally termed premature senescence, even though morphologically both premature and replicative senescent cells appear similar. When DNA damage checkpoints are intact, the senescence phenotype can be maintained irreversibly for a long period of time. Cell cycle checkpoint activities involving p53/p21 and $\mathrm{pRB} / \mathrm{p} 16$ play critical roles in initiation and maintenance of the senescence state [7]. When these cell cycle checkpoints proteins are inactivated, cells bypass or overcome senescence and continue to proliferate at the expense of genomic stability leading to genetic instability and in most instances to cell death (instead of senescence). Rare survivor cells $\left(10^{-5}-10^{-7}\right)$ escaping from this crisis period acquire the ability to maintain telomere length, in most cases this is accomplished by upregulating or reactivating telomerase. Rarely, a smaller fraction of cells can escape senescence and crisis by maintaining telomeres by a DNA recombination pathway termed alternative lengthening of telomeres (ALT) [8].

At the cellular level, senescence is generally believed to 
be a biological mechanism operating to suppress oncogenic conversion of normal cells. Consistent with the two-step hypothesis for senescence and immortalization [7], It has been experimentally shown that senescence is not only a potent defense against cancer [9-14], but that cancer cells that have escaped senescence can be reversed to senescence by restoration of the tumor suppressor $\mathrm{p} 53[15,16]$. It is thus widely believed that senescence and immortalization share some common biology, and telomeres and telomerase may be the connection [17].

Telomerase is necessary for the long-term proliferation potential of human stem cells and cancer cells, and for normal tissue renewal. Ectopic expression of telomerase in normal human cells leads to extension of life-span or immortalization of many cell types $[18,19]$. Inhibition of telomerase in telomerase positive cancer cells can lead to the induction of cell death [20-22]. Finally, mutations in either $h T R$ or TERT are associated with many premature aging phenotypes leading to human diseases [23-25]. Moreover, telomerase knockout mice and hTERT transgenic mice provided compelling evidence of critical roles of telomerase in aging and cancer [26]. To date, the only defined activity of telomerase is the elongation and the maintenance of telomeres. However, increasing evidence is emerging to implicate telomerase in aging and cancer not only by maintaining telomeres, but by the actions beyond the chromosome ends (e.g. independent of telomere main- tenance). The precise molecular mechanism of telomerase functions independent of telomere maintenance is becoming a broader question. In this review, we will discuss the recent progress in understanding the roles of telomerase in several important cellular processes other than telomere length maintenance (Figure 1).

\section{Telomerase in apoptosis}

Inhibition of telomerase by targeting $h T R$ or $h T E R T$ that induce apoptosis has been widely investigated and considered to be a promising strategy in cancer treatment [27]. The explanation for the link between inhibition of telomerase and apoptosis has been logically taken into account of the role of telomerase in telomere maintenance. Thus, inhibition of telomerase results in progressive telomere shortening or inactivation of the telomere capping function of the shelterin complex and this eventually leads to cell death.

However, accumulating evidence suggests that telomerase has a role in apoptosis regulation independent of its classical function in telomere maintenance. It has been shown that the catalytic subunit of telomerase hTERT contains a mitochondrial localization signal peptides at its $\mathrm{N}$-terminal 20 amino acids which targets hTERT to mitochondria, resulting in active telomerase in this organelle [28]. These experiments were done by transiently over-expression of

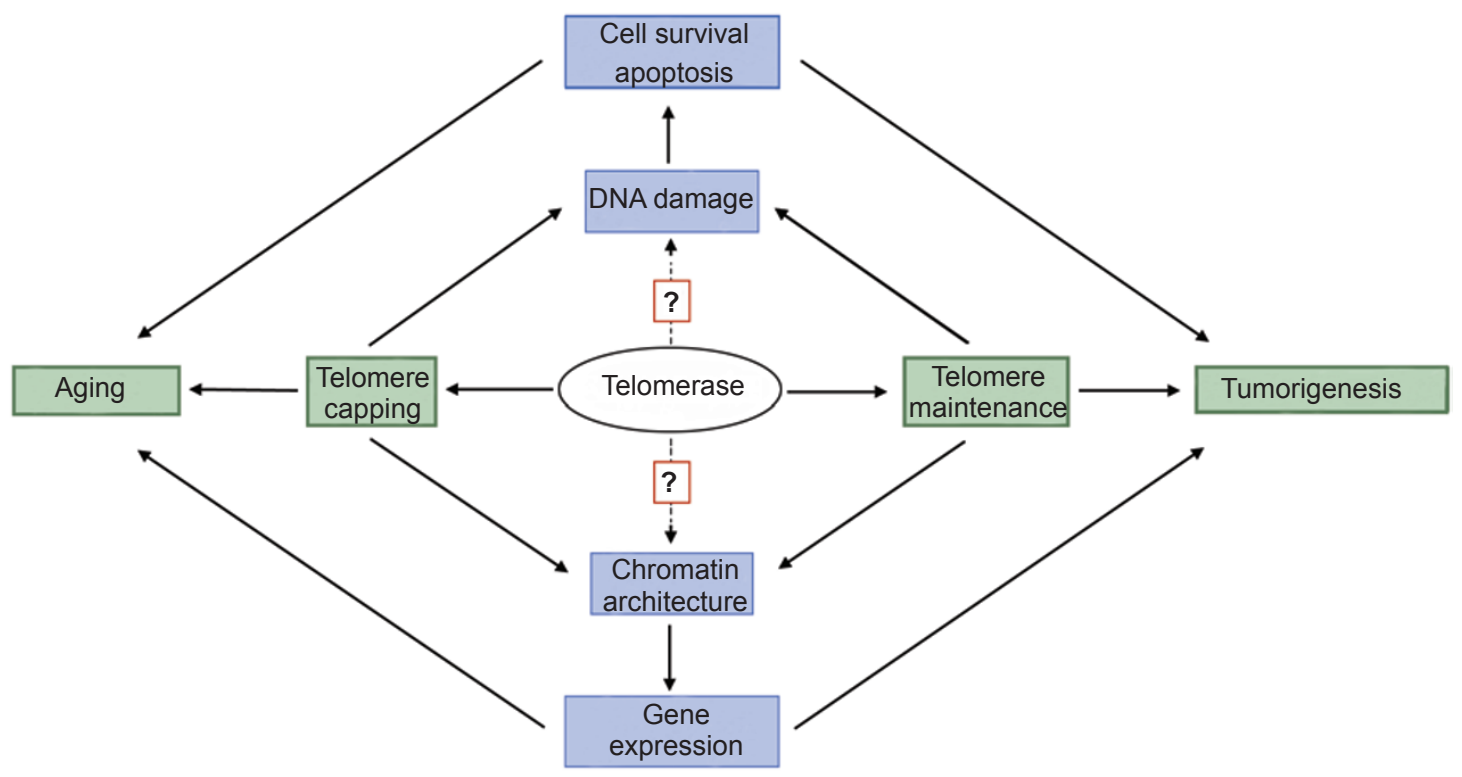

Figure 1 Potential pathways of telomerase actions link to aging and cancer. Telomerase maintains telomere integrity by elongating telomere length and capping telomeres. Additionally, telomerase may involve directly or indirectly in DNA damage response, in regulation of chromatin architecture and in regulation of gene expression, collectively contributing to aging and cancer. 
the green fluorescent protein GFP fused with either fulllength hTERT or the first 20 amino acids in seven different cell lines. In all case tested, a mitochondrial localization of GFP-hTERT was observed by confocal microscopy and by the telomeric repeat amplification protocol (TRAP). Therefore, the mitochondrial localization of hTERT was not cell type specific. However, due to the lack of highly specific antibodies against hTERT and low expression of endogenous hTERT in cells, it has not been examined whether endogenous hTERT localizes in mitochondria under physiological condition.

Further analysis shows that the mitochondrial telomerase sensitizes the damage effects of $\mathrm{H}_{2} \mathrm{O}_{2}$ on mtDNA, presumably via modulation of metal homeostasis [28]. In addition, studies indicate that mitochondrial localization of telomerase functions is a determinant of hydrogen peroxide-induced mitochondrial DNA damage and apoptosis [29]. Mutations in the hTERT N-terminal mitochondrial leader sequence resulted in loss of mitochondrial targeting without affecting the catalytic activity of telomerase. Cells expressing these mutated $h T E R T$ exhibited reduced level of mitochondrial DNA damage induced by hydrogen peroxide treatment [29]. These observations of proapoptosis activity of hTERT in mitochondria are consistent with the experiments showing that oxidative stress induces the nuclear export of hTERT, which might contribute to the mitochondrial proapoptosis activity of hTERT [30]. However, molecular mechanisms of hTERT targeting into mitochondria and precise roles of hTERT in mitochondria remain to be investigated. Identification of mitochondrial proteins that interacts with hTERT will be helpful to address these questions.

In contrast, earlier studies have demonstrated that overexpression of hTERT rendered cells more resistant to apoptosis. For example, expression of telomerase does not prevent stress-induced senescence in normal human fibroblasts but protects the cells from apoptosis and necrosis [31]. In addition, it has been shown that overexpression of $h T E R T$ suppresses apoptosis at a premitochondrial step before the release of cytochrome $\mathrm{c}$ and apoptosis-inducing factor [32]. Recent studies showed that down-regulation of $h T E R T$ expression by $h T E R T$ specific interfering RNA (siRNA) sensitizes mitochondrial pathways of apoptosis without apparent involvement of telomere erosion due to telomerase inhibition, rather by post-translational activation of Bax protein which triggers a CD-90 independent mitochondrial pathway of apoptosis [33]. More recent report indicated that TERT promotes cellular and organismal survival independent of telomerase activity [34]. In this report, Lee et al have demonstrated by using both cell cultures and a transgenic mouse model that expression of TERT was directly associated with increased resistance to apoptosis induced by staurosporine (STS) or N-methyl-
D-aspartic acid (NMDA) treatment. Importantly, these investigators have shown that the antiapoptotic effect of TERT was independent of telomere maintenance and telomerase activity [34].

Thus it appears that hTERT may exhibit distinct functions in apoptosis regulation: promoting apoptosis by altering mitochondrial membrane potential or metal homeostasis in mitochondria, while inhibiting apoptosis by interacting with repair pathway in the nucleus. Modulation of apoptosis by hTERT may provide new strategies for cancer chemotherapy applications. The detailed molecular mechanisms of hTERT in apoptosis regulations clearly merit additional investigation.

\section{Telomerase in DNA damage responses}

The link between telomeres and DNA damage was the initial observation that led to the discovery of the special property of chromosome ends that protect against chromosome fusions. It's been now well documented that the telomeres are closely linked to the DNA damage response machinery. Many DNA repair proteins such as Mre11/ Rad50/NBS, Ku, DNA PKcs, BLM/WRN and ERCC1/XPC have been found to be associated with telomeres [35-37]. Senescent cells exhibit distinct DNA foci at dysfunctional telomeres that contain DNA damage response proteins such as 53BP1, Rad17, H2AX, Mre11 and ATM [38-41]. This suggests that the dysfunctional telomeres provoke the general DNA damage response machinery to mediate and maintain the irreversible G1 arrest in senescent cells. However, recent studies suggest telomerase itself may be involved in DNA damage responses independent of its role in telomere maintenance. It has been demonstrated that telomerase is transiently expressed in S-phase in some normal human cells, yet fails to maintain overall telomere length, but protect karyotypic stability by "capping" chromosome ends and by resetting chromatin during DNA replication [42] without adding telomeric repeats. An earlier report indicated that intrachromosomal DNA breaks are occasionally repaired by healing with telomeric DNA repeat sequences [43], suggesting that telomerase may participate in DNA repair. In addition, hTERT has been found to be associated with DNA replication protein primase [44]. As proteins involved in DNA replication are usually required for DNA repair, this observation indicates a possible role of telomerase in DNA repair functions. Furthermore, the catalytic subunit of telomerase hTERT has been found to be physically associated with telomeres and DNA repair proteins, thereby enhancing genomic stability and DNA repair functions [45]. It has also been shown that ectopic expression of hTERT leads to increased expression of genes involving in DNA damage repair and changes in 
the interaction of the telomeres with the nuclear matrix inside the cell nucleus. This is associated with reduction of spontaneous chromosome damage in G1 cells, enhancement of the kinetics of DNA repair [46]. Collectively, these observations suggest a role of telomerase in DNA damage response some of which may be due to functions independent of telomere maintenance.

There is now emerging evidence that telomerase regulates overall chromatin states and DNA damage responses [47]. In normal diploid human fibroblasts, suppression of the telomerase catalytic subunit expression by $h T E R T$ shRNA impairs the cellular response to DNA double-strand breaks induced by ionizing radiation without apparent telomere shortening and altering telomere integrity. This has been demonstrated by introduction of wild type as well as hTERT mutants that retain catalytic activity, but have a defect in telomere maintenance in vivo, yet restore DNA damage responses, although less efficiently. It is known that the ATM protein undergoes rapid autophosphorylation upon exposure of ionizing radiation or chromatin modification reagents such as trichostatin A (TSA). In cells that lack hTERT expression, ATM autophosphorylation induced by TSA treatment is significantly impaired. In addition, chromatin in cells lacking hTERT was more susceptible to micrococcal nuclease digestion, suggesting the suppression of $h T E R T$ expression alters the overall chromatin architecture [47].

It appears that involvement of telomerase in DNA repair responses is not restricted to DNA double-strand breaks, but to a more broad range of DNA repairs mechanisms including nucleotide excision repair capacity [48]. However, several other studies found no correlation between telomerase expression and DNA repair capacity $[49,50]$. In summary, direct molecular evidence explaining the involvement of telomerase in DNA repair activity is still lacking. Thus, a testable experimental model to examine telomere independent activity of telomerase in DNA damage responses and repair functions is clearly needed.

Intriguingly, recent studies showed that ectopic expression of $h T E R T$ in human mammary epithelial cells resulted in reduced basal level of active p53, and other p53 dependent signaling [51]. Based on these observations, it was proposed that the nontelomeric functions of telomerase may be explained through its direct effects on short telomeres and indirect effects on p53 dependent signaling [52]. For instance, it is possible that some telomeres become short during serial passages while the bulk of telomeres are long, ectopic expression of $h T E R T$ elongates the short telomeres, and consequently stops or reduces the p53 signaling and therefore provides cells a growth advantage. However this model may not explain all activities and consequences of hTERT expression. For example, ALT cells without telom- erase maintain their telomeres by telomerase-independent pathway, and expression of oncogenic H-Ras in the immortal ALT cell line GM847 fails to induce their transformation. However, subsequent expression of hTERT results in their tumorigenic conversion [53]. Furthermore, it has been shown that overexpression of TERT induced proliferation of hair follicle stem cells in a transgenic mouse model [54, 55]. Significantly, induced proliferation of quiescent stem cells by TERT overexpression does not require the RNA component of telomerase, again suggesting that hTERT may have telomere-maintenance independent functions. In addition, telomerase was found to localize in and to shuttle from different compartments within cells, depending on the cell-cycle stage, transformation and state of overall DNA damage [56]. Therefore, telomerase may be involved in different cell signaling pathways when targeted to different locations or when responding to different stimulations, such as in DNA damage responses and tumorigenesis.

\section{Telomerase in stem cells}

Telomerase activity is extinguished in a majority of adult tissues after birth, whereas several adult stem cell compartments still maintain low levels of telomerase activity but insufficient to prevent progressive telomere shortening with age [57]. However the presence of telomerase in these adult stem cell populations is believed to be important for stem cell function and organism fitness. Increasing evidence suggests that aging and cancer share many common aspects of biology and are considered as stem cell diseases resulting from decline or defect in regenerative capacity and organ homeostasis. Thus, telomerase may be the connection of these two fundamental biological processes [7]. Mutations in telomerase components are associated with several diseases of premature aging characterized by bone marrow failure, likely through impairment in stem cell functions caused by telomerase deficiencies. Studies from telomerase deficient mice models and human age-related diseases caused by mutations in telomerase components have documented that telomere shortening is a determinant of the ability of stem cell function, tissue homeostasis and perhaps organismal aging. Insufficient or defects in telomerase activity in stem cell compartment provoke telomere shortening that results in loss of cells and in tissue dysfunction [58]. Interestingly, regardless of the telomere length in species, knocking out of telomerase in mice, Caenorhabditis elgans and plants such as Arabidopsis results in reproduction failure after several generations in addition to other abnormalities [59-61]. Recent studies have demonstrated a function of telomerase in activating stem cells through a mechanism that does not required telomerase activity. Using either a constitutive [54] or inducible [55] 
transgenic mouse that over-expresses mTERT (the catalytic subunit of mouse telomerase), it has been demonstrated that telomerase over-expression activates epidermal stem cells (ESC), and promotes ESC mobilization upon treatment with proliferation stimuli. This induced ESC mobilization was accompanied by increased keratinocyte proliferation, enhanced hair growth and augmented skin hyperplasia. Importantly, these studies showed the promoting effect on hair growth by TERT over-expression in a mouse strain that is deficient in the RNA component of telomerase [54] or by TERT catalytic mutant [55]. Therefore these studies convincingly demonstrated that in addition to its established role in extending telomeres, TERT has its own activity in stem cell behavior independent of telomerase activity and telomere maintenance. More recent observations by Artandi and co-workers provides further support for a novel function of TERT in activating stem cells independent of telomerase activity [62]. They showed that TERT mutants lacking reverse transcriptase (RT) function retains the full activities in activating resting hair follicle stem cells and in enhancing keratinocyte proliferation in mouse skin, thereby inducing initiation of a new hair follicle growth phrase and promotes hair synthesis [62]. This study demonstrated that functions of TERT in the synthesis of telomere repeats and the novel function in stem cell behavior are genetically distinct. Furthermore, in order to understand the mechanism of the RT-independent function for TERT, Artandi and co-workers investigated the genome-wide transcriptional response to acute changes in TERT levels in mouse skin by a large scale microarray analysis [62]. They showed that acute withdrawal of TERT causes a rapid change in expression of genes involved in epithelial development, signal transduction and cytoskeleton/adhesion. Interestingly, statistical analysis using pattern matching algorithms revealed that the TERT transcriptional response strongly resembles those mediated by Myc and Wnt, two important factors in stem cell function and cancer. This finding suggested that the role of TERT in activating stem cells may be mediated by transcriptional regulation of a developmental program converging on the Myc and Wnt pathways.

Recently, several groundbreaking studies show that differentiated human somatic cells can be reprogrammed into a pluripotent state known as induced pluripotent stem cells (iPS) by transduction of four defined transcription factors (Oct4, Sox2, Kfl4 and Myc) [63-65]. Human iPS cells were similar to human embryonic stem (ES) cells in morphology, proliferation, surface antigens, gene expression, epigenetic status of pluripotent cell-specific genes, and expression of telomerase activity. Significantly, Park et al. showed that transduction of $h T E R T$ and gene of SV40 large $\mathrm{T}$ in addition of the four transcription factors results in a higher frequency of human iPS colony formation [65].
This observation suggested hTERT may have a supportive role in iPS cells formation.

Together, although telomere maintenance is a determinant for stem cell functionality and tissue fitness, nontelomeric mechanism of telomerase in stem cell behavior may also contribute to functionality of stem cells in tissue homeostasis and organimal aging in vivo. The mechanism may act by telomerase or hTERT itself on stem cell function independent of telomere maintenance remains a challenging question. Regulation in global gene expression profiles or epigenetic modifications induced by hTERT or telomerase as suggested by Artandi and co-workers [62] and as discuss below warrants further investigations.

\section{Telomerase in the regulation of gene expression}

Several microarray analyses indicate that telomerase has a regulatory role in gene expression. (a) Ectopic expression of telomerase in human mammary epithelial cells results in upregulation of five growth promoting genes and downregulation of seven growth inhibitory genes [66]. These correlated with a diminished requirement for exogenous mitogen and enhanced cell proliferation. (b) Global expression profiling experiments in bovine adrenocortical cells immortalized by $h T E R T$ showed that a total of 284 genes were affected by $h T E R T$ expression, and the function of these genes involves in diverse group of cellular processes ranging from cell cycle regulation, metabolism, differentiation, apoptosis and cell signaling [67]. (c) The comparison of the gene expression profiles revealed that 172 genes differentially expressed between normal human fibroblasts and $h T E R T$ immortalized fibroblasts, one of the highly expressed genes in $h T E R T$ immortalized fibroblasts encoded epiregulin, a potent growth factor [68]. (d) Gene profiling studies by targeting mTER in murine melanoma B16 cell line showed down-regulation of 138 genes, eight of which are involved in the glycolytic pathway. Significantly, changes in gene expression, particularly genes encoding glycolytic enzymes establish a functional link between telomerase and cancer metastasis [69]. (e) Targeting $h T R$ in human colon cancer cell line HCT116 resulted in downregulation of genes implicated in angiogenesis and metastasis [70]. Other studies have found that hTERT up-regulates a secreted tumor antigen Mac-2 binding protein (Mac-2BP) that is overexpressed in many cancers and implicated in tumor metastasis [71], and that hTERT also up-regulates cyclin D1 expression in prostate cancer cells [72]. The biological significance of these observations implicates novel molecular mechanisms of telomerase functions in many cellular processes by targeting genes and pathways besides its well defined functions in telomere biology.

The broader spectrum changes in gene expression pro- 
files affected by telomerase suggest a role of telomerase in epigenetic regulation and in modulation of chromatin architecture, Changes in chromatin could consequently affect different set of genes in different cellular contexts and states. In support of this hypothesis, it has been observed that overexpression of $h T E R T$ up-regulated and stabilized the DNA 5-methylcytosine transferase I in normal human fibroblasts [73]. In addition, suppression of $h T E R T$ has been shown to alter the overall configuration of chromatin [47]. It is well known that the epigenetic mechanisms play crucial roles in the repression of telomerase expression in normal human cells and in the activation of telomerase expression during immortalization and transformation. Conversely, telomerase may participate in epigenetic regulation by telomere maintenance and regulation of telomere states, or by interacting with chromatin modifier factors; thereby eliciting it's telomere-dependent and -independent activities in gene expression regulation, as well as in apoptosis and DNA damage responses. It is also possible that telomerase acts as an effector of transcription by interacting directly with certain transcriptional factors or signaling pathways. In support of this hypothesis, it has been shown that hTERT interacts directly with the nuclear factor NF- $\mathrm{BB}$ p65 in vivo and this interaction mediates nuclear translocation of telomerase [74]. It remains to be elucidated whether the complex of hTERT-NF- $\kappa$ B p 65 can be recruited to the promoter of genes regulated by hTERT.

Interestingly, it seems that there are some feedback loop regulations between genes targeting $h T E R T$ and hTERT expression itself. For examples, (a) earlier studies demonstrated that the oncoprotein c-Myc activates $h T E R T$ expression and telomerase activity [75]. Conversely, hTERT immortalized human mammary epithelial cells show up-regulation of c-Myc expression [76]. (b) Similarly, several tumor suppressors including $\mathrm{pRB}$ and $\mathrm{p} 53$ negatively regulate $h T E R T$ expression and repress telomerase activity [6]. However, overexpression of hTERT in human lens epithelial cell lines results in down-regulation of p21, p53 expression, as well as hyperphosphorylation of $\mathrm{pRB}$ and up-regulation of E2F transcriptional activity [77]. And suppression of $h T E R T$ expression also results in elevated p53 and p21 transcription [78]. (c) The histone methyltransferase (SMYD3) implicated in oncogenesis directly transactivates the $h T E R T$ promoter and is required for inducible and constitutive $h T E R T$ expression in normal and malignant human cells [79]. On the other hand, it has been shown that expression of hTERT up-regulated transcription and promoter activity of DNA 5-methylcytosine transferase I gene (DNMT1) in normal human fibroblasts [73], although the mechanism by which hTERT-induced DNMT1 expression has yet to be determined. These interplays between telomerase and tumor suppressor proteins at the transcriptional level may contribute to the functions of telomerase in immortalization and tumorigenesis beyond the chromosome ends.

\section{Concluding remarks}

The central role of telomere/telomerase biology in aging and cancer has been well documented by using cellular and animal models. However, pathways and molecular mechanisms by which telomerase provokes aging and cancer are still far from being fully understood. There are some findings that telomere maintenance independent activities of telomerase as discussed in this review might provide additional insights into the mechanisms of actions of telomerase in aging and cancer (Figure 1). The question that remains unclear is whether the involvement of telomerase in cellular process other than telomere maintenance, such as apoptosis, DNA damage response, tumorigenesis, and gene expression control, requires the telomerase holoenzyme containing $h T R$ and hTERT, or hTERT alone. While there are some persuasive experiments indicating that telomerase may "moonlight" many of the telomere-maintenance independent functions proposed for telomerase, many of these observations may indeed still be related to telomeres. In summary, there is mounting evidence that hTERT has different roles when it associates with different factors or targets to different locations away from telomeres and new functions of telomerase are starting to be elucidated.

\section{Acknowledgments}

Research in author's lab was supported in part by a grant from the National Natural Science Foundation of China (No. 30671065), the Research Fund for the Doctoral Program of High Education (No. 20060027008), and the National Important Basic Research Project (No. 2007CB507402) to Yusheng Cong. Support from NASA grants NNJ06HD92G and NNJ05HD36G (JWS) is acknowledged.

\section{References}

1 Müller HJ. The remaking of chromosomes. Collecting Net 1938; 13:181-198.

2 McClintock B. The stability of broken ends of chromosomes in Zea mays. Genetics 1941; 26:234-282.

3 De lange T, Lundblad V and Blackburn EH. Telomeres. 2nd Edition. Cold Spring Harbor Laboratory Press, 2006.

4 Blackburn EH. Telomere states and cell fates. Nature 2000; 408:53-56.

5 Collins K. The biogenesis and regulation of telomerase holoenzymes. Nature reviews Mol Cell Biol 2006; 7:484-494.

6 Cong YS, Wright WE, Shay JW. Human telomerase and its regulation. Microbiol Mol Biol Rev 2002; 66:407-425.

7 Shay JW, Wright WE. Senescence and immortalization: role of 
telomeres and telomerase. Carcinogenesis 2005; 26:867-874.

8 Stewart SA, Weinberg RA. Telomeres: cancer to human aging. Annu Rev Cell Dev Biol 2006; 22:531-557.

9 Wu C, Miloslavskaya I, Demontis S, Maestro R, Galaktionov $\mathrm{K}$. Regulation of cellular response to oncogenic and oxidative stress by Seladin-1. Nature 2004; 432:640-645.

10 Michaloglou C, Vredeveld LC, Soengas MS, et al. BRAF ${ }^{\mathrm{E} 600}$-associated senescence-like cell cycle arrest of human naevi. Nature 2005; 436:720-724.

11 Collado M, Gil J, Efeyan A, Guerra C et al. Senescence in premalignant tumours. Nature 2005; 436:642.

12 Braig M, Lee S, Loddenkemper C, et al. Oncogene-induced senescence as an initial barrier in lymphoma development. Nature 2005; 436:660-665.

13 Chen Z, Trotman LC, Shaffer D, et al. Crucial role of p53dependent cellular senescence in suppression of Pten-deficient tumorigenesis. Nature 2005; 436:725-730.

$14 \mathrm{Wu}$ CH, van Riggelen J, Yetil A, Fan AC, Bachireddy P, Felsher DW. Cellular senescence is an important mechanism of tumor regression upon c-Myc inactivation. Proc Natl Acad Sci USA 2007; 104:13028-13033.

15 Xue W, Zender L, Miething C, et al. Senescence and tumour clearance is triggered by p53 restoration in murine liver carcinomas. Nature 2007; 445:656-660.

16 Ventura A, Kirsch DG, McLaughlin ME, et al. Restoration of p53 function leads to tumour regression in vivo. Nature 2007; 445:661-665.

17 Finkel T, Serrano M, Blasco MA. The common biology of cancer and ageing. Nature 2007; 448:767-774.

18 Bodnar AG, Ouellette M, Frolkis M, et al. Extension of life-span by introduction of telomerase into normal human cells. Science 1998; 279:349-352.

19 Harley CB. Telomerase is not an oncogene. Oncogene 2002; 21:494-502.

20 Hahn WC, Stewart SA, Brooks MW, et al. Inhibition of telomerase limits the growth of human cancer cells. Nat Med 1999; 10:1164-1170.

21 Herbert B, Pitts AE, Baker SI, et al. Inhibition of human telomerase in immortal human cells leads to progressive telomere shortening and cell death. Proc Natl Acad Sci USA 1999; 96:14276-14281.

22 Zhang X, Mar V, Zhou W, Harrington L, Robinson MO. Telomere shortening and apoptosis in telomerase-inhibited human tumor cells. Genes Dev 1999; 13:2388-2399.

23 Artandi SE. Telomerase, and Human Disease. $N$ Engl J Med 2006; 355:1195-1197.

24 Yamaguchi H, Calado RT, Ly H, et al. Mutations in hTERT, the gene for telomerase reverse transcriptase, in aplastic anemia. $N$ Engl J Med 2005; 352:1413-1424.

25 Tsakiri KD, CronkhiteJT, Kuan PJ, et al. Adult-onset pulmonary fibrosis caused by mutations in telomerase. Proc Natl Acad Sci USA 2007; 104:7552-7557.

26 Blasco MA. Mice with bad ends: mouse models for the study of telomeres and telomerase in cancer and aging. EMBO J 2005; 24:1095-1103.

27 Shay JW, Wright WE. Telomerase therapeutics for cancer: challenges and new directions. Nat Rev Drug Discov 2006; 7:577584 .

28 Santos JH, Meyer N, Skorvaga M, et al. Mitochondrial hTERT exacerbates free-radical-mediated mtDNA damage. Aging Cell 2004; 3:399-411.

29 Santos JH, Meyer JN, Van Houten B. Mitochondial localization of telomerase as determinant for hydrogen peroxide-induced mitochondrial DNA damage and apoptpsis. Hum Mol Genet 2006; 15:1757-1768

30 Haendeler J, Hoffmann J, Diehl JF, et al. Antioxidants inhibit nuclear export of telomerase reverse transcriptase and delay replicative senescence of endothelial cells. Circ Res 2004; 94:768-775.

31 Gorbunova V, Seluanov A, Pereira-Smith OM. Expression of human telomerase (hTERT) does not prevent stress-induced senescence in normal human fibroblasts but protects the cells from stress-induced apoptosis and necrosis. J Biol Chem 2002; 277:38540-38549.

32 Zhang P, Chan SL, Fu W, Mendoza M, Mattson MP. TERT suppresses apoptotis at a premitochondrial step by a mechanism requiring reverse transcriptase activity and 14-3-3 protein-binding ability. FASEB J 2003; 17:767-769.

33 Massard C, Zermati Y, Pauleau AL, et al. hTERT: a novel endogenous inhibitor of the mitochondrial cell death pathway. Oncogene 2006; 25:4505-4514.

34 Lee J, Sung YH, Cheong C, et al. TERT promotes cellular and organismal survival independently of telomerase activity. Oncogene 2008 Jan 28; [Epub ahead of print].

35 de Lange T. Shelterin: the protein complex that shapes and safeguards human telomeres. Genes Dev 2005; 19:2100-2110.

36 Meier A, Fiegler H, Munoz P, et al. Spreading of mammalian DNA-damage response factors studied by ChIP-chip at damaged telomeres. EMBO J 2007; 26:2707-2718.

37 Verdun RE, Karlseder J. The DNA damage machinery and homologous recombination pathway act consecutively to protect human telomeres. Cell 2006; 127:709-720.

38 Sedelnikova OA, Horikawa I, Zimonjic DB, Popescu NC, Bonner WM, Barrett JC. Senescing human cells and ageing mice accumulate DNA lesions with unrepairable double-strand breaks. Nat Cell Biol 2004; 6:168-170.

39 d'Adda di Fagagna F, Reaper PM, Clay-Farrace L, et al. A DNA damage checkpoint response in telomere-initiated senescence. Nature 2003; 426:194-198.

40 Takai H, Smogorzewska A, de Lange T. DNA damage foci at dysfunctional telomeres. Curr Biol 2003; 13:1549-1556.

41 Narita M, Nunez S, Heard E, et al. Rb-mediated heterochromatin formation and silencing of E2F target genes during cellular senescence. Cell 2003; 113:703-716.

42 Masutomi K, Yu EY, Khurts S, et al. Telomerase maintains telomere structure in normal human cells. Cell 2003; 114:241-253.

43 Stellwagen AE, Haimberger ZW, Veatch JR, Gottschling DE. Ku interacts with telomerase RNA to promote telomere addition at native and broken chromosome ends. Genes Dev 2003; 17:23842395.

44 Ray S, Karamysheva Z, Wang L, Shippen DE, Price CM. Interactions between telomerase and primase physically link the telomere and chromosome replication machinery. Mol Cell Biol 2002; 22:5859-5868.

45 Sharma GG, Hwang KK, Pandita RK, et al. Human heterochromatin protein 1 isoforms HP1(Hsalpha) and HP1(Hsbeta) interfere with hTERT-telomere interactions and correlate with changes in cell growth and response to ionizing radiation. $\mathrm{Mol}$ 
Cell Biol 2003; 23:8363-8376.

46 Sharma GG, Gupta A, Wang H, et al. hTERT associates with human telomeres and enhances genomic stability and DNA repair. Oncogene 2003; 22:131-146.

47 Masutomi K, Possemato R, Wong JM, et al. The telomerase reverse transcriptase regulates chromatin state and DNA damage responses. Proc Natl Acad Sci USA 2005; 102:8222-8227.

48 Shin KH, Kang MK, Dicterow E, Kameta A, Baluda MA, Park $\mathrm{NH}$. Introduction of human telomerase reverse transcriptase to normal human fibroblasts enhances DNA repair capacity. Clin Cancer Res 2004; 10:2551-2560.

49 Kampinga HH, Van Waarde-Verhagen MA, Van Assen-Bolt AJ, et al. Reconstitution of active telomerase in primary human foreskin fibroblasts: effects on proliferative characteristics and response to ionizing radiation. Int J Radiat Biol 2004; 80:377-388.

50 Bates SE, Zhou NY, Federico LE, Xia L, O'Connor TR. Repair of cyclobutane pyrimidine dimers or dimethylsulfate damage in DNA is identical in normal or telomerase-immortalized human skin fibroblasts. Nucleic Acids Res 2005; 33:2475-2485.

51 Beliveau A, Bassett E, Lo AT. P53-dependent integration of telomere and growth factor deprivation signals. Proc Natl Acad Sci USA 2007; 104:4431-4436.

52 Beliveau A. Soothing the watchman: Telomerase reduces the p53-dependent cellular stress response. Cell Cycle 2007; 6:12841287.

53 Stewart SA, Hahn WC, O'Connor BF, et al. Telomerase contributes to tumorigenesis by a telomere length-independent mechanism. Proc Natl Acad Sci USA 2002; 99:12606-12611.

54 Sarin KY, Cheung P, Gilison D, et al. Conditional telomerase induction causes proliferation of hair follicle stem cells. Nature 2005; 436:1048-1052.

55 Flores I, Cayuela ML, Blasco MA. Effects of telomerase and telomere length on epidermal stem cell behavior. Science 2005; 309:1253-1256.

56 Wong JM, Kusdra L, Collins K. Subnuclear shuttling of human telomerase induced by transformation and DNA damage. Nat Cell Biol 2002; 4:731-736.

57 Collins K, Mitchell JR. Telomerase in the human organism. Oncogene 2002; 21:564-579.

58 Blasco MA. Telomere length, stem cells and aging. Nat Chem Biol 2007; 3:640-649.

59 Rudolph KL, Chang S, Lee HW, et al. Longevity, stress response, and cancer in aging telomerase-deficient mice. Cell 1999; 96:701712.

60 Riha K, McKnight TD, Griffing LR, Shippen DE. Living with genome instability: plant responses to telomere dysfunction. Science 2001; 291:1797-1800.

61 Cheung I, Schertzer M, Rose A, Lansdorp PM. High incidence of rapid telomere loss in telomerase-deficient Caenorhabditis elegans. Nucleic Acids Res 2006; 34:96-103.

62 Choi J, Southworth LK, Sarin KY, et al. TERT promotes epithelial proliferation through transcriptional control of a Myc- and Wnt-related developmental program. PLoS Genet 2008; 4:e10.
Epub 2007 Dec 13.

63 Takahashi K, Tanabe K, Ohnuki M, et al. Induction of pluripotent stem cells from adult human fibroblasts by defined factors. Cell 2007; 131:861-872.

$64 \mathrm{Yu}$ J, Vodyanik MA, Smuga-Otto K, et al. Induced pluripotent stem cell lines derived from human somatic cells. Science 2007; 318:1917-1920.

65 Park IH, Zhao R, West JA, et al. Reprogramming of human somatic cells to pluripotency with defined factors. Nature 2008; 451:141-146.

66 Smith LL, Coller HA, Roberts JM. Telomerase modulates expression of growth-controlling genes and enhances cell proliferation. Nat Cell Biol 2003; 5:474-479.

67 Perrault SD, Hornsby PT, Betts DH. Global gene expression response to telomerase in bovine adrenocortical cells. Biochem Biophys Res Commun 2005; 335:925-936.

68 Lindvall C, Hou M, Komurasaki T, et al. Molecular characterization of human telomerase reverse transcriptase-immortalized human fibroblasts by gene expression profiling: activation of the Epiregulin gene. Cancer Res 2003; 63:1743-1747.

69 Bagheri S, Nosrati M, Li S, et al. Genes and pathways downstream of telomerase in melanoma metastasis. Proc Natl Acad Sci USA 2006; 103:11306-11311.

70 Li S, Crothers J, Haqq CM, Blackburn EH. Cellular and gene expression responses involved in the rapid growth inhibition of human cancer cells by RNA interference-mediated depletion of telomerase RNA. J Biol Chem 2005; 280:23709-23717.

71 Park YP, Choi SC, Kim JH, et al. Up-regulation of Mac-2 binding protein by hTERT in gastric cancer. Int J Cancer 2007; 120:813820.

72 Jagadeesh S, Banerjee PP. Telomerase reverse transcriptase regulates the expression of a key cell cycle regulator, cyclin D1. Biochem Biophys Res Commu 2006; 347:774-780.

73 Yonng JI, Sedivy JM, Smith JR. Telomerase expression in normal human fibroblasts stabilizes DNA 5-methylcytosine transferase I. J Biol Chem 2003; 278:19904-19908.

74 Akiyama M, Hideshima T, Hayashi T, et al. Nuclear Factor- $\kappa \mathrm{B}$ p65 mediates tumor necrosis factor $\alpha$-induced nuclear translocation of telomerase reverse transcriptase protein. Cancer Res 2003; 63:18-21.

75 Wang J, Xie LY, Allan S, Beach D, Hannon GJ. Myc activates telomerase. Genes Dev 1998; 12:1769-1774.

76 Wang J, Hannon GJ, Heach DH. Risky immortalization by telomerase. Nature 2000; 405:755-756.

77 Xiang H, Wang J, Mao Y, et al. Human telomerase accelerates growth of lens epithelial cells through regulation of the genes mediating RB/E2F pathway. Oncogene 2002; 21:3784-3791.

78 Lai SR, Cunningham AP, Huynh VQ, et al. Evidence of extra-telomeric effects of hTERT and its regulation involving a feedback loop. Exp Cell Res 2007; 313:322-330.

79 Liu C, Fang X, Ge Z, et al. The telomerase reverse transcriptase (hTERT) gene is direct target of histone methyltransferase SMYD3. Cancer Res 2007; 67:2626-2631. 